\title{
FRACTURA DE CLAVÍCULA Y DISOCIACIÓN CLÍNICO-RADIOLÓGICO EN EL RECIÉN NACIDO: REPORTE DE UN CASO
}

\author{
Custodio Olsen Quispe Condori ${ }^{1,2}$
}

\begin{abstract}
RESUMEN
La fractura de clavícula es la lesión ósea más frecuente del recién nacido. Los factores de riesgo son: obstétricos, maternos y fetales. Las manifestaciones clínicas de la fractura de clavícula se hacen evidentes al segundo y tercer día de vida, su diagnóstico requiere de una confirmación radiológica. A veces la radiografía solicitada no contribuye a confirmar el diagnóstico de fractura de clavícula, a pesar del cuadro clínico evidente. Esto se conoce como la disociación clínico radiológico. El manejo de fractura de clavícula debe iniciarse tan pronto como se haya hecho el diagnóstico clínico y tener presente que placa radiográfica es un examen auxiliar, es decir, no siempre se va a apreciar la lesión ósea. Es importante valorar la pericia del evaluador en la toma de decisiones. Se presenta un caso de un recién nacido de parto eutócico con fractura de clavícula y disociación clínico radiológico. El presente trabajo realizado en el Instituto Nacional Materno Perinatal, tiene por objetivo contribuir al conocimiento médico e intercambiar experiencias en el diagnóstico y manejo de un recién nacido con fractura de clavícula y disociación clínico radiológico.
\end{abstract}

Palabras clave: Fractura de clavícula; Traumatismos obstétricos (Fuente: DeCS BIREME).

\section{CLAVICLE FRACTURE AND DISSOCIATION CLINICAL RADIOLOGICAL IN THE NEWBORN: CASE REPORT}

\begin{abstract}
The clavicle fracture is the most common bone lesion of the newborn. The risk factors are: obstetric, maternal and fetal. The clinical manifestations of the clavicle fracture are more evident at the second and third day of life his diagnostic requires of one radiological confirmation; sometimes the requested radiography does not contribute to confirm the diagnosis of fracture of clavicle despite evident clinical picture. This is known as the clinical radiological dissociation. The management of fractured clavicle should be started as soon as you have done the clinical diagnosis and keep in mind that the radiographic plate is an auxiliary examination that is to say not always going to appreciate the bone lesion. It is important to assess the expertise of the evaluator in the decision making. A case is presented of a newborn eutocic delivery with clavicle fracture and dissociation clinical radiological. The present work realized in the Instituto Nacional Materno Perinatal by objective to contribute to medical knowledge and exchanging experiences in the diagnosis and management a newborn with fracture clavicle and dissociation clinical radiological.
\end{abstract}

Key words: Clavicle fracture; Birth trauma (Source: MeSH NLM).

\section{INTRODUCCIÓN}

La clavícula es un hueso plano, lisa casi en toda su extensión, con forma de "S" itálica, situado debajo de la piel y del músculo platisma, en la parte anterosuperior del tórax, junto con la escápula forman la cintura escapular. Se puede palpar por toda su longitud y se extiende del esternón al acromion de la escápula, siguiendo una dirección oblicua lateral y posterior; presenta dos caras (superior e inferior), dos bordes (anterior y posterior) y dos extremidades (acromial y esternal) ${ }^{1}$.

La fractura de clavícula es la lesión ósea más frecuente del recién nacido, probablemente por su característica anatómica, ocurre en la mayor parte de las veces en el tercio medio y la incidencia de fracturas de clavícula se ha utilizado como un indicador de calidad asistencial obstétrica $^{2,3}$.
Cuadro clínico: a la inspección suele estar presente la disminución de la movilidad del miembro superior afecto, asimetría de las fosas claviculares debido a la protrusión o abombamiento de la zona de lesión ósea por la formación del hematoma; a la palpación superficial irregularidad en el trayecto de la clavícula afecta comparado con el lado sano 4,5. Desde el momento de la sospecha clínica de fractura de clavícula, se debe aplicar los principios de la bioética para no manipular ni realizar movimientos del miembro superior afecto, tampoco realizar la palpación profunda buscando el signo de la tecla, el crujido o la crepitación de la clavícula comprometida, ni realizar el reflejo de moro, a fin de no aumentar el dolor que ya lo tiene ${ }^{6}$.

En el diagnóstico de la fractura de clavícula interviene la pericia del que explora. Los factores de riesgo pueden estar relacionadas a: factores maternos como la primiparidad, baja estatura, anomalías pélvicas; a factores obstétricos

Servicio de Puericultura, Departamento de Neonatología, del Instituto Nacional Materno Perinatal.

Departamento Académico de Pediatría de la Facultad de Medicina de la Universidad Nacional Mayor de San Marcos. Lima-Perú. 
como la presentación podálica, desproporción feto pélvica, parto precipitado, parto dificultoso y prolongado; y a factores del feto como la macrosomía fetal y anomalías fetales ${ }^{4,5,7}$. Como examen auxiliar solicitar una radiografía o una ecografía para confirmar el diagnóstico de fractura de clavícula ${ }^{3,5}$. A veces, la radiografía solicitada no contribuye al diagnóstico clínico de fractura de clavícula. Este hecho se conoce como la disociación clínico radiológico y posiblemente, la frecuencia real de la disociación clínico radiológico sea desconocida y mayor que las descritas al tratarse de una lesión infra diagnosticada ${ }^{3,4,8}$. Frente a un caso de fractura de clavícula con disociación clínico radiológico, iniciar cuanto antes el manejo de fractura de clavícula como son la inmovilización del miembro superior afecto e indicar los analgésicos. El pronóstico en general es bueno y las complicaciones: pocas veces se asocia a lesiones neurológicas, vasculares, a lesiones pleuro pulmonares, pseudoartrosis ${ }^{4,5}$. El presente trabajo realizado en el Instituto Nacional Materno Perinatal, tiene por objetivo contribuir al conocimiento médico e intercambiar experiencias en el diagnóstico y manejo de un recién nacido (RN) con fractura de clavícula y disociación clínico radiológico. La madre del $\mathrm{RN}$ dio su consentimiento para la presente publicación.

\section{CASO CLÍNICO}

RN de parto eutócico, trabajo de parto espontáneo, presentación cefálica, no sufrimiento fetal, no analgesia de parto, no recibió drogas, primer período trabajo de parto 6 horas y $45 \mathrm{~min}$, segundo periodo de 18 minutos, con maniobra de kristeller, ruptura de membranas de 48 horas, líquido amniótico normal, cordón umbilical normal, ocurrido en Sala de Partos del Instituto Nacional Materno Perinatal, el día 23 de setiembre del año 2013, a las 11,03 horas, de sexo masculino, peso de $3090 \mathrm{~g}$, talla de 49 $\mathrm{cm}$, perímetro cefálico de $34 \mathrm{~cm}$, perímetro torácico de 33 , cm y Apgar normal.

Datos maternos: Madre de 27 años, conviviente, con tercer año de educación superior, seis controles prenatales, dos de psico profilaxis, sin antecedentes de enfermedades familiares, enfermedades maternas ni infecciones maternas, peso preconcepcional $41 \mathrm{Kg}$, peso actual $53 \mathrm{Kg}$, talla $149 \mathrm{~cm}$, paridad G2 P0010. Ingresa la paciente por el Servicio de Emergencia el día 22-09-2 013 a las 05,00 horas. Exámenes auxiliares de laboratorio, de Presión Arterial y Temperatura dentro de los valores referenciales. Ecografía: 22-09-2 013, de 37 sem, ponderado fetal 3353 g. Índice de líquido amniótico $6 \mathrm{~cm} 3$.

Examen físico al nacer: buen estado general, cara, cuello, clavícula, tórax y otros normales.

Diagnóstico al nacer: RN de término varón de 39 semanas, Adecuado para la Edad Gestacional. Tiene indicaciones de pasar al Servicio de Alojamiento conjunto, con lactancia materna exclusiva.
Evolución: Visita médica a las 20 horas de vida, el RN está junto con su madre, cuyo examen físico fue normal.

Visita médica a las 44 horas de vida, en el examen físico a la inspección se apreció leve disminución de movilidad de miembro superior derecho, asimetría de las fosas claviculares, por leve abombamiento de la fosa clavicular derecha; a la palpación superficial se percibió leve irregularidad en el trayecto de la superficie de la clavícula derecha, hechos que no fueron percibidos por la madre ni en la visita médica anterior. Impresión diagnóstica: fractura de clavícula derecha. Indicaciones: inmovilizar el miembro superior derecho y solicitud de radiografía de clavículas.

A las 48,00 horas de vida, la radiografía de clavículas (figura 1), no contribuyó a confirmar el diagnóstico de la fractura de clavícula. Indicaciones: reevaluación preferencial del examen físico de clavículas, en la visita médica del día siguiente, por probable disociación clínico radiológico. Indicaciones continuar la inmovilización del miembro superior derecho y paracetamol como analgésico. Se informó a los padres.

Visita médica a las 70 horas de vida, describe el cuadro clínico encontrado el día anterior, y solicitan una nueva radiografía de clavículas.

A las 72 horas de vida, es evaluado con la segunda radiografía (figura 2 ), donde se observa lesión clavicular derecha, confirmándose la fractura de clavícula derecha. Indicaciones: continuar el manejo de fractura de clavícula derecha. Se informó a los padres y se pidió el consentimiento para publicar el presente trabajo.

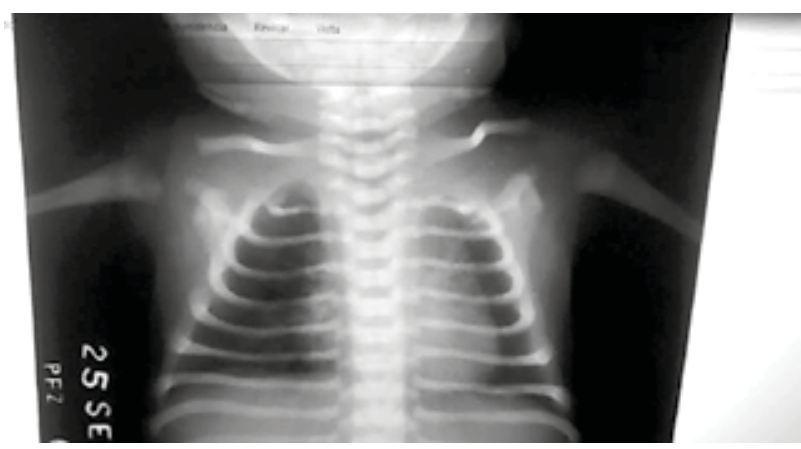

Figura 1. Primera radiografía no se aprecia la lesión ósea clavícula derecha

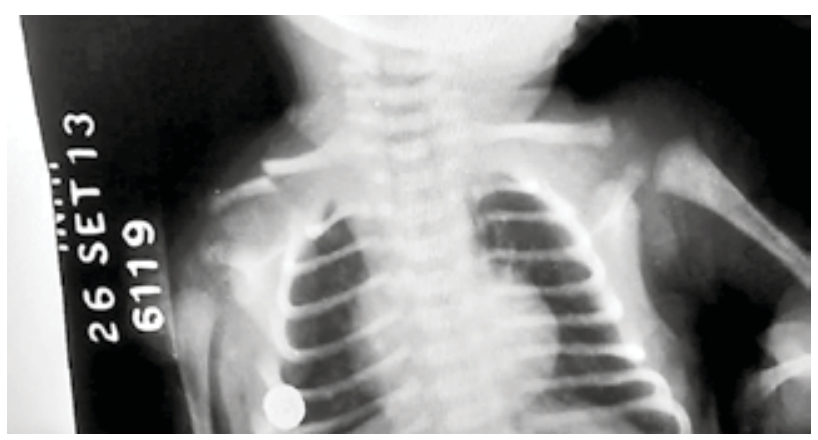

Figura 2. Segunda radiográfica se aprecia la lesión ósea clavícula derecha. 


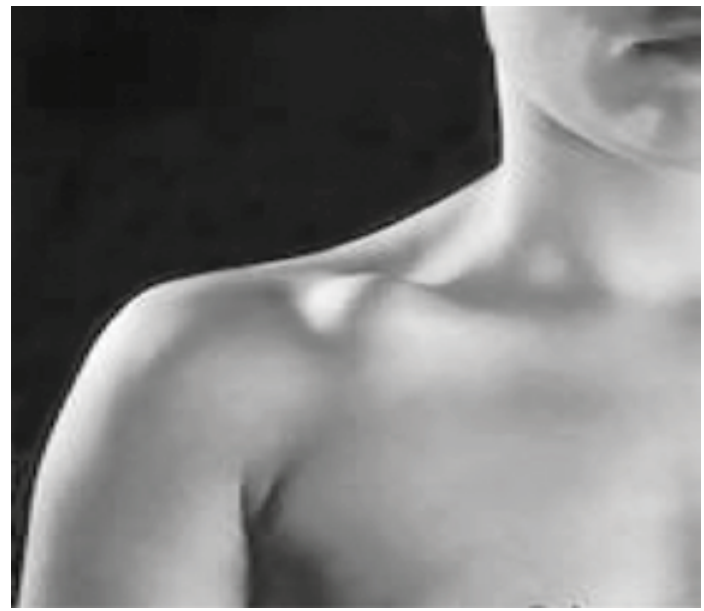

Figura 3. Defecto estético como secuela de fractura de clavícula derecha.

\section{DISCUSIÓN}

En el presente caso las evaluaciones médicas de las clavículas al nacer y a las 20 horas de vida fueron normales. A las 44 horas de vida se apreciaron las manifestaciones clínicas de lesión ósea clavicular derecha ${ }^{4,5}$, la que no es confirmada por la radiografía ${ }^{3}$; sin embargo, el RN recibió las indicaciones como si se tratara de una fractura de clavícula ${ }^{9}$ A las 72 horas de vida, la fractura de clavícula es confirmada radiológicamente. Al respecto la mayor parte de las fracturas de clavículas se diagnostican en el Servicio de Alojamiento Conjunto, el segundo día de vida, que también es la segunda evaluación médica del neonato, en el examen físico a la inspección se observó disminución de la movilidad del miembro superior derecho con asimetría de las fosas claviculares por la protrusión o abombamiento en la fosa clavicular derecha, por la formación del hematoma en el lugar de la lesión vascular ósea. A la palpación superficial se percibió una irregularidad en el trayecto de la clavícula derecha. La magnitud del hematoma de la lesión ósea se manifiesta después de horas de vida, dependiendo del tipo de lesión ósea ${ }^{4,5,9}$. En el RN motivo de estudio la fractura de clavícula se sospechó por el cuadro clínico a las 44 horas de vida y recibió las indicaciones del manejo de una fractura de clavícula, a pesar de que la placa radiográfica no contribuyó para su diagnóstico. A las 72 horas de vida, fue evaluado con la segunda radiografía (figura 2), donde se observa lesión clavicular derecha, confirmándose la fractura de clavícula y recibió las indicaciones de continuar con el manejo de fractura de clavícula. Frente a una fractura de clavícula con disociación clínico radiológico, el manejo de la fractura de clavícula debe iniciarse tan solo con el diagnóstico clínico ${ }^{3,9}$. Conviene comentar lo siguiente: al ver la radiografía de clavículas no solo apreciar las partes óseas, sino también hay que apreciar las partes blandas, como buscar o ver la imagen del hematoma de fractura de clavícula producida por la lesión ósea, dicho hematoma no aparece en el momento de ocurrir la lesión, esta se manifiesta con el transcurso de las horas, como al día siguiente, en la Figura 1, se puede apreciar la asimetría de las partes blandas de las fosas claviculares, en el lado derecho se observa una imagen irregular alrededor de la lesión ósea, que corresponde al hematoma de fractura de clavícula. La presencia de la imagen del hematoma corrobora la lesión ósea. En el presente caso, el parto fue atendido en horas de la mañana de un día laborable, por personal calificado y no en otro momento, como para pensar que fue atendido por personal con menos experiencia ${ }^{10}$. Como también hay estudios que concluyen que la mayoría de las fracturas de clavículas se presenta en RN de trabajo de parto y parto normal y los factores de riesgo de lesión ósea clavicular no ofrecen como un medio de predicción clínica prenatal ${ }^{2,11}$.

La consolidación de la fractura de clavícula ocurre en dos a tres semanas, dependiendo del tipo de lesión ósea. El pronóstico fue bueno y no tuvo complicaciones ${ }^{4,5,11}$. La información a la madre y familia son importantes, muchas veces causa un impacto emocional desagradable, tanto para quién lo recibe como también para quién lo comunica, impacto que varía de persona a persona, debido a varios factores como el grado de instrucción, su participación en el parto, la duración del parto.

Conclusión: el caso presentado es un RN de parto eutócico que en su evolución al segundo día de vida se hace el diagnóstico de fractura de clavícula con disociación clínico radiológico, recibiendo desde entonces las indicaciones del manejo de fractura de clavícula. Se recomienda a los profesionales que atienden a los RN con diagnóstico clínico de fractura de clavícula indicar el manejo correspondiente y no descontinuar el manejo si la placa radiográfica no contribuye a su diagnóstico ${ }^{3,9}$, a fin de disminuir secuelas a largo plazo como el defecto estético de la zona clavicular afecta, tal como se puede apreciar en la figura 3 .

Fuente de financiamiento: recursos propios.

Conflicto de intereses: ninguno

\section{REFERENCIAS BIBLIOGRÁFICAS}

1. Latarjet RL, Ruiz A. Anatomía Humana. $4^{\mathrm{a} e d}$. Editorial Médica Panamericana S. A. Buenos Aires. Argentina. 2004. p. 471-472.

2. Lurie S, Wand S, Golan A, Sadan O. Risk factors for fractured clavicle in the newborn. Journal of Obstetrics and Gynaecology Research. November 2011; 37 (11):1572-1574.

3. Beall MH, Ross MG. Clavicle Fracture in Labor: Risk Factors and Associated Morbidities. Journal of Perinatology 2001; 21: 513-515.

4. Archilla B, Engels V, San Frutos, Toro P, Bueno B, Bajo JM, y col. Fracturas de clavícula en el neonato: incidencia y factores de riesgo. Prog Obstet Ginecol 2004; 47(3):113-8.

5. Reiners $\mathrm{CH}$, Souid AK, Oliphant M, Newman N. Palpable Spongy Mass Over the Clavicle, an Underutilized Sign of Clavicular Fracture in the newborn. Clin Pediatr (Phila) 2000; 39: 695-698.

6. Siurana JC. Ëtica de las decisiones clínicas ante pacientes incapaces. Veritas 2006;1 (15): 223-244. 
7. Linder I, Melamed N, Kogan A, Merlob P, Yogev Y, Glezerman $M$. Gender and birth trauma in full-term infants. The Journal of Maternal-Fetal and Neonatal Medicine 2012; 25(9): 1603-1605.

8. Kayser R, Mahlfeld K, Heyde C, Grasshoff H. Ultrasonographic imaging of fractures of the clavicle in newborn infants. Journal of Bone and Joint Surgery. Jan 2008; 85 (1):115-116.

9. Reed MJ, Fernandez-Dias N. The missed clavicle fracture in children? Emerg Med J. July 2006; 23(7):581-583.

10. Linder N, Linder I. Fridman E, Kouadio F, Lubin D, Merlob P, et al. Birth trauma-risk factors and short-term neonatal outcome. The Journal of Maternal-Fetal and Neonatal Medicine 2013; 26(15): 1491-1495.
11. Kaplan B, Rabinerson D, Avrech OM, carmi N, Steinberg DM, Merlob P. Fracture of the clavicle in the newborn following normal labor and delivery. International Journal of Gynecology \& Obstetrics 1998; 63:15-20.

Correspondencia: Custodio Olsen Quispe Condori

Dirección: Jirón Juan Bujanda $N^{\circ} 335$, Departamento $N^{\circ} 504$, Urbanización Montecarmelo, la Victoria

Teléfono: 6395434/ 999909896

Correo electrónico: custodioolsen@hotmail.com 